\title{
The Effect of Luteolin and Luteoloside on the Secondary Structure of Lysozyme
}

\author{
Yu Wu, ${ }^{1}$ Lijian Cui, ${ }^{2}$ Lingling Qu, ${ }^{1}$ Rong Wang, ${ }^{1}$ Ning Chen, ${ }^{1}$ Yun Huang $\mathbb{D}^{1}$, \\ Yan Zhang $\mathbb{D}^{1,3}$ and Pin $L v \mathbb{D}^{1}$ \\ ${ }^{1}$ Hebei Medical University, Shijiazhuang 050017, Hebei, China \\ ${ }^{2}$ Experiment Center, Hebei University of Chinese Medicine, Shijiazhuang 050020, Hebei, China \\ ${ }^{3}$ Hebei Food Inspection and Research Institute, Hebei Food Safety Key Laboratory, Shijiazhuang 050227, Hebei, China
}

Correspondence should be addressed to Yun Huang; 17400921@hebmu.edu.cn, Yan Zhang; snowwinglv@126.com, and Pin Lv; lvpinzy@163.com

Received 20 January 2021; Revised 25 March 2021; Accepted 3 April 2021; Published 23 April 2021

Academic Editor: Biao Yuan

Copyright ( 92021 Yu Wu et al. This is an open access article distributed under the Creative Commons Attribution License, which permits unrestricted use, distribution, and reproduction in any medium, provided the original work is properly cited.

\begin{abstract}
The changes of lysozyme conformation in the absence and presence of luteolin and luteoloside were investigated by spectral analysis including fluorescence, UV, CD, Raman, and ATR-FTIR, and the biological activity of lysozyme was investigated by lysozyme assay kit. The results showed that the microenvironment hydrophobicity of lysozyme increased and peptide extension decreased with the addition of luteolin or luteoloside. The $\alpha$-helix of lysozyme might be influenced by luteolin or luteoloside, and its relative content had a significant difference after adding luteolin or luteoloside by the ATR-FTIR method, which was reconfirmed by CD and Raman spectra. The lysozyme activity changed obviously after adding luteolin or luteoloside. All of the conclusions above indicated the active site of lysozyme in the $\alpha$-helix might be influenced by luteolin and luteoloside.
\end{abstract}

\section{Introduction}

Lysozyme (LYSO), also called muramidase, killing or inhibiting the bacteria with little drug resistance, is an important immune factor widely existing in many tissues of the human body $[1,2]$. It can lyse cell walls of bacteria [3], without degradation to the cells in the human body. As early as 2010, the National Health Commission of P. R. China issued a document allowing lysozyme to be used as a food additive. In addition, lysozyme is clinically available to promote the repair of injured tissues, to correct the degree of immune activation, and to reduce the acute inflammatory reaction, especially in burn wound infection and herpes treatment [4]. Lysozyme has a globular fold divided into two domains, whose native conformation displays four $\alpha$-helix structures and a three-strand antiparallel $\beta$-sheet [5] which consist of 129 amino acid residues, including six Trp residues, three of which (Trp 62, Trp 63, and Trp 108) locate in the active pocket of the helices domain of lysozyme [6]. Therefore, it is very meaningful to research the change of helices domain structure which is closely associated with the activity of lysozyme. Flavonoids exhibit a wide range of physiological activity $[7,8]$, which have specific nutritional and medicinal values with antioxidant, antiviral, and antibacterial activities, such as luteolin and its glycoside (luteoloside, luteolin-7-O-glucoside) (Figure 1) widely distributed in vegetables, fruits, and some food-medicine herbs including pepper, chrysanthemum indicum, honeysuckle, and perilla, approved by the National Ministry of Health. Although lysozyme, luteolin, or luteoloside has many biological activities with little drug resistance, the biological activity of them used alone is limited. Our previous studies [9] have shown that some flavonoids can form noncovalent complexes with lysozyme, so it is very necessary to explore whether this combination would have any influence on the structure-activity of lysozyme.

The synchronous fluorescence spectrum is used to research the information about the microenvironment in the vicinity of the chromophores, which has several advantages including high sensitivity, simplicity, and reducing the influence of spectral overlap and diffused light [10]. The shift of the maximum emission wavelength can prove the change of 


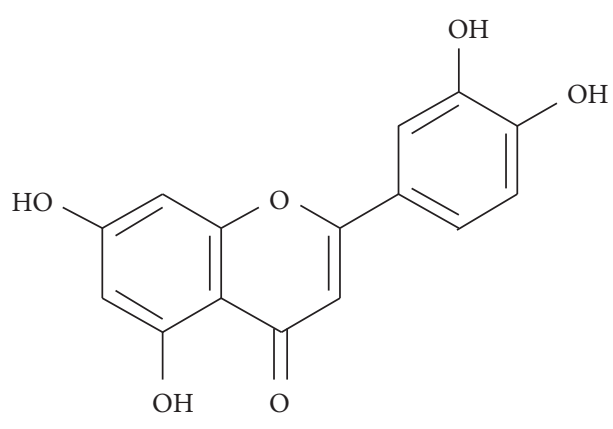

(a)

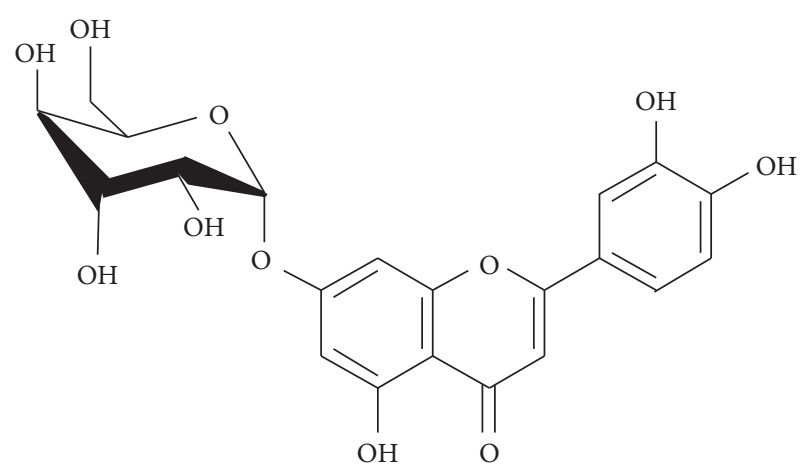

(b)

Figure 1: Structures of luteolin (a) and luteoloside (b).

amino residues microenvironment polarity of lysozyme in the absence and presence of luteolin or luteoloside, which is related to the lysozyme conformation [11].

UV absorption spectrum shows the characteristic peaks of the peptide bond, tryptophan residues, and tyrosine residues at about 210 and $280 \mathrm{~nm}$ [12].

Circular dichroism (CD) spectrum [13], Raman spectrum [14], and Fourier transform infrared reflection (FTIR) $[15,16]$ spectrum belong to molecular vibration spectra, which are usually used to investigate the secondary structure change of protein. The different types of secondary structure contained in protein produce the characteristic CD spectra in the far UV [17], primarily from 190 to $230 \mathrm{~nm}$, in which two negative peaks at about $220 \mathrm{~nm}$ and $208 \mathrm{~nm}$ caused by a negative Cotton effect are the main characteristic peaks of $\pi-\pi *$ and $n-\pi *$ transitions of peptide bonds in lysozyme $\alpha$-helix. In Raman and infrared spectra, we primarily focus on the amide I $\left(1700-1600 \mathrm{~cm}^{-1}\right)$ and the amide III $\left(1220-1330 \mathrm{~cm}^{-1}\right)$ bands, which are sensitive to the secondary structure of protein, caused by $\mathrm{C}=\mathrm{O}$ stretching, $\mathrm{C}-\mathrm{N}$ stretching, and $\mathrm{N}-\mathrm{H}$ in-plane bending vibration [18]. Raman spectrum is important to study protein molecular structure, which overcomes water peak interference and weak signal of some chromophores [19]. The ranges of $1650-1660 \mathrm{~cm}^{-1}$ and $1260-1300 \mathrm{~cm}^{-1}$ are suitable for the analysis of lysozyme $\alpha$-helix [20], for which the shift and intensities of peaks are sensitive. The change of $\alpha$-helix structure of lysozyme can be further inferred by combining with FTIR spectra and then speculating the change of its active site.

FTIR with the attenuated total reflection (ATR) attachment can provide the important structures' information of lysozyme solution. The absorption bands observed in the amide I region correspond to $\alpha$-helix $\left(1642-1660 \mathrm{~cm}^{-1}\right)$, $\beta$-sheet $\left(1613-1637 \mathrm{~cm}^{-1}\right.$ and $\left.1682-1689 \mathrm{~cm}^{-1}\right), \quad \beta$-turn $\left(1662-1700 \mathrm{~cm}^{-1}\right)$, and disordered $\left(1637-1645 \mathrm{~cm}^{-1}\right)$ structures [21]. In cooperation with the information from $\mathrm{CD}$ and Raman, comparing the changes of lysozyme secondary structure relative contents in the absence and presence of luteolin and luteoloside by ATR-FTIR can infer the change of lysozyme active site.

The structure of lysozyme is related to the biological activity and its change may influence the lysozyme biological function. There are many methods [22-24] to detect lysozyme activity, including turbidimetry, colorimetry, and agar plate method, among which turbidimetry is the most widely used in the detection with rapidness high reliability, and simple operation. As a classical method, turbidimetry is also popularized by Pharmacopoeia Commission of PRC. To confirm whether the lysozyme activity was influenced by flavonoids, we did the lysozyme activity test with the lysozyme assay kit.

Our previous studies have indicated that when small molecule compounds combined with lysozyme, lysozyme usually increased their biological activities, such as lysozyme enhancing the antibacterial activity of baicalin, baicalein, and scutellarin and increasing the cytotoxicity of caffeine to HepG2 cells. But the influence of the combination on the biological activity of lysozyme is still unclear. This paper focuses on the study of the nonspecific binding of luteolin or luteoloside to lysozyme to evaluating their application from the perspective of lysozyme activity, which might provide important theoretical research for the attempt of combined utilization between lysozyme and luteolin or luteoloside in the field of food and health.

\section{Materials and Methods}

2.1. Materials and Spectral Equipment. Lysozyme was purchased from China Pharmaceutical Group Chemical Reagent Co., Ltd. Luteolin and luteoloside were from the National Institute for the Control of Pharmaceutical and Bioproducts (China). Lysozyme assay kit $(30 \mathrm{~T} / 28 \mathrm{~S})$ was bought from the Nanjing Jiancheng Bioengineering Institute.

All spectra were mainly measured on F-380 spectrofluorometer (China), TU-1901 UV-Vis spectrophotometer (Purkinje general, China), MOS-450 spectropolarimeter (Bio-Logic, French), DXR SmartRaman Spectrometer (Thermo, Germany), and FTIR-8400S spectrophotometer (Shimadzu, Japan) with the ATR attachment (PIKE, USA).

2.2. Procedures and Methods. Appropriate amounts of lysozyme were dissolved in Tris-HCl buffer solution to $1.0 \times 10^{-2} \mathrm{mmol} / \mathrm{L}$ as lysozyme stock solution. Luteolin or luteoloside solutions were dissolved with $50 \%$ methanol- 
Tris-HCl buffer solution to $1.0 \mathrm{mmol} / \mathrm{L}$ as stock solution. All the stock solutions were diluted to the lower concentrations for actual use with Tris- $\mathrm{HCl}$ buffer solution.

2.2.1. Fluorescence Spectrum Analysis. A $2.0 \mathrm{~mL}$ solution containing $1.0 \mu \mathrm{mol} / \mathrm{L}$ lysozyme, was titrated by successive additions of $1.0 \mathrm{mmol} / \mathrm{L}$ luteolin or luteoloside. The final concentration of luteolin or luteoloside was $6.0 \mu \mathrm{mol} / \mathrm{L}$ at an increment of $1.0 \mu \mathrm{mol} / \mathrm{L}$.

Fluorescence spectra were recorded in the range of $290-350 \mathrm{~nm}$ with an excitation wavelength of $280 \mathrm{~nm}$. The synchronous fluorescence spectra were obtained by scanning the excitation and emission monochromator simultaneously. The wavelength interval was set at $60 \mathrm{~nm}$. Both excitation and emission bandwidths were adjusted at $5 \mathrm{~nm}$.

2.2.2. UV Spectrum Analysis. A $2.0 \mathrm{~mL}$ solution, containing $1.0 \mu \mathrm{mol} / \mathrm{L}$ lysozyme, was titrated by successive additions of $1.0 \mathrm{mmol} / \mathrm{L}$ luteolin or luteoloside. The final concentration of luteolin or luteoloside was $1.0 \mu \mathrm{mol} / \mathrm{L}$.

UV spectra of lysozyme, luteolin, luteoloside, luteolinlysozyme, and luteoloside-lysozyme were recorded in the range of 200-400 nm. The UV differential spectrum of LYSO is obtained by subtracting the spectrum of luteolin or luteoloside solution from that of LYSO in the presence of luteolin or luteoloside.

2.2.3. CD Spectrum Analysis. Mos- $450+$ SMF-300 spectrometer was used to scan between 185 and $250 \mathrm{~nm}$. The optical length of the sample cell was $1 \mathrm{~mm}$, scan speed $100 \mathrm{~nm} / \mathrm{min}$, and resolution $0.1 \mathrm{~nm}$. Each sample was measured three times. The final concentration of lysozyme was $1.0 \times 10^{-2} \mathrm{mmol} / \mathrm{L}$ in the presence or absence of $3.0 \times 10^{-1} \mathrm{mmol} / \mathrm{L}$ luteolin or luteoloside.

2.2.4. Raman Spectrum Analysis. Raman spectrum of lysozyme solution containing $200 \mu \mathrm{L}$ lysozyme stock solution $\left(1.0 \times 10^{-2} \mathrm{mmol} / \mathrm{L}\right)$ and $220 \mu \mathrm{L}$ Tris- $\mathrm{HCl}$ buffer solution was recorded in the range of $1200-1800 \mathrm{~cm}^{-1}$. Another solution, containing $400 \mu \mathrm{L}$ Tris- $\mathrm{HCl}$ and $20 \mu \mathrm{L}$ luteolin or luteoloside stock solution, was used for the determination of flavonoid Raman spectrum. The last solution, containing $200 \mu \mathrm{L} \mathrm{ly-}$ sozyme stock solution, $200 \mu \mathrm{L}$ Tris- $\mathrm{HCl}$, and $20 \mu \mathrm{L}$ luteolin or luteoloside stock solution, was used for the determination of lysozyme-flavonoid Raman spectrum. All the above measurements were carried out at room temperature with 200 scanning times. The interference of the background solvent should be deducted before the sample is determined. Spectra processing procedures were followed: spectra of Tris-HCl buffer solution and lysozyme solution were collected first, and then the spectrum of Tris- $\mathrm{HCl}$ buffer solution was subtracted from that of lysozyme solution to obtain the differential spectrum of lysozyme. We used the same technique to obtain the differential spectrum of lysozyme after binding with luteolin (or luteoloside) by lysozyme-luteolin (or luteoloside) minus luteolin (or luteoloside).
2.2.5. ATR-FTIR Spectrum Analysis. FTIR absorption spectra were taken by the ATR-FTIR method with the resolution of $4 \mathrm{~cm}^{-1}$ and 30 scans. The solutions used for the determination of the infrared spectrum were prepared in the same way as Raman spectrum analysis. The spectrum was measured without pressure and the IR beam was directly onto the crystal and the internal reflectance created the evanescent wave getting through the sample [25]. Spectra processing procedures were similar to those of Raman spectrum analysis.

The FTIR differential spectra were first to be baseline correction and then were smoothed with the five-point Savitzky-Golay smooth function through the Origin 9.0. The second-derivative spectra were assigned to the different types of lysozyme secondary structures. Fourier selfdeconvolution with the secondary derivative was used to estimate the number, position, and width of bands from 1700 to $1600 \mathrm{~cm}^{-1}$ in the amide I region [26].

2.2.6. Lysozyme Activity Assay. The lysozyme assay kit was applied to measure the lysozyme activity. The content of the active lysozyme was calculated according to the assay kit instructions. Put $0.2 \mathrm{~mL}$ distilled water and $2.0 \mathrm{~mL}$ bacterial working solution into the control tube. $0.2 \mathrm{~mL} 2.5 \mu \mathrm{g} / \mathrm{mL}$ standard lysozyme working solution and $2.0 \mathrm{~mL}$ bacteria working solution were added into the standard tube. The sample lysozyme solutions contained $1.0 \times 10^{-2} \mathrm{mmol} / \mathrm{L}$ lysozyme with $0,1 \times 10^{-2}, 2 \times 10^{-2}, 3 \times 10^{-2}, 4 \times 10^{-2}$, and $5 \times 10^{-2} \mathrm{mmol} / \mathrm{L}$ luteolin or luteoloside. $0.2 \mathrm{~mL}$ sample solution and $2.0 \mathrm{~mL}$ bacteria working solution were put into the sample tube. The above tubes were placed in $37^{\circ} \mathrm{C}$ water bath for 15 minutes and then put in the ice water bath for 3 minutes. We transferred them in a cuvette with $1 \mathrm{~cm}$ light path and measured the $T_{15}$ values at $530 \mathrm{~nm}$ by using the UV-Vis measurement which was adjusted $100 \%$ transmittancy with the distilled water and then calculated the contents of active lysozyme by the equation followed.

Active lysozyme $=\left(\mathrm{UT}_{15}-\mathrm{OT}_{15}\right) \times$ lysozyme standard concentration $(2.5 \mu \mathrm{g} / \mathrm{mL}) \times$ dilution ratio of sample $/\left(\mathrm{ST}_{15}-\mathrm{OT}_{15}\right)$, in which $\mathrm{UT}_{15}$ was the transmittancy of sample tube after 15minute water bath, $\mathrm{OT}_{15}$ was that of control group, and ST15 standard tube. The activity of standard lysozyme is regarded as 1.

2.2.7. Data Analysis. The data were processed in SPSS 13.0, which performed the independent-samples $t$-test to compare the difference. If $P$ values $<0.05$, it was considered to have significant differences.

\section{Results and Discussion}

The spectra of proteins are closely related to the microenvironments of luminescent amino acid residues. When proteins combine with endogenous or exogenous substances, they usually show changes in spectral behaviors. Therefore, spectrometry is commonly used to study the interaction between proteins and small molecules. 
3.1. Fluorescence Spectra of Luteolin-Lysozyme System and Luteoloside-lysozyme System. Trp 62 and Trp 108, located at the substrate binding sites, are the main fluorophores in LYSO, whose maximum emission wavelength is closely associated with the microenvironment [27]. The synchronous spectra give the characteristic information of Trp residues when $\Delta \lambda$ was set at $60 \mathrm{~nm}$. The maximum emission wavelength of Trp residues did blue shifts, indicating the microenvironment hydrophobicity increased and the spread of peptide strands decreased [28]. The fluorescence intensities of lysozyme decreased to 396 regularly with the increasing concentration of luteolin (a2 in Figure 2), and 385 with luteoloside (b2 in Figure 2). Meanwhile, from the synchronous fluorescence spectra of LYSO-luteolin (a1 in Figure 2) and LYSO-luteoloside (b1 in Figure 2) when $\Delta \lambda=60 \mathrm{~nm}$, luteolin made the emission peak blue-shift $4 \mathrm{~nm}$ from $286 \mathrm{~nm}$ to $282 \mathrm{~nm}$ and luteoloside did $2 \mathrm{~nm}$ from 285 to $283 \mathrm{~nm}$. Both luteolin and luteoloside could increase hydrophobicity and decrease the polarity of the microenvironment around Trp, but luteolin had more influence on the microenvironment with a more obvious blue shift than luteoloside did. In addition, the lysozyme activity might be influenced, because the Trp residues located in the active site of lysozyme.

3.2. UV Spectra. The difference of peak intensities and shifts in the UV differential spectra of luteolin (or luteoloside)LYSO-luteolin (or luteoloside), UV absorption spectrum of LYSO, and those of luteolin (or luteoloside)-LYSO system at about $210 \mathrm{~nm}$ and $280 \mathrm{~nm}$ suggested the complex formation between luteolin (or luteoloside) and lysozyme.

The addition of luteolin or luteoloside made the absorption peaks of LYSO increase in the luteolin (or luteoloside)-LYSO system (Figure 3), which has two main peaks [29] (208 and $278 \mathrm{~nm}$ ) in its absorption spectrum, showing that the peak intensity at 208 increased slightly without shift and the peak at $278 \mathrm{~nm}$ increased $18 \%$ with blue shift $3 \mathrm{~nm}$ or $8 \mathrm{~nm}$ in the presence of luteolin or luteoloside. Compared with absorption spectra of LYSO, the peak intensity at $208 \mathrm{~nm}$ in UV differential spectra decreased $6 \%$ or $8 \%$ without shift and the peak at $278 \mathrm{~nm}$ decreased slightly without shift or decreased $25 \%$ with blue shift $2 \mathrm{~nm}$.

3.3. Lysozyme Secondary Structure Change. The active site of lysozyme is mainly in the helices region, so we further focus on studying the change of lysozyme $\alpha$-helix. The spectroscopic methods have shown that lysozyme conformation changed with the addition of luteolin or luteoloside. The hydroxyl group on the benzene ring of flavonoid might combine with - NH-CO- in lysozyme to change the stretching vibration of $\mathrm{C}=\mathrm{O}$, and the hydrogen on the hydroxyl group of benzene ring might form a hydrogen bond with $\mathrm{C}-\mathrm{N}$ in lysozyme, changing the stretching vibration of $\mathrm{C}-\mathrm{N}$, which increased lysozyme microenvironment hydrophobicity and made the peptide chain structure more compact. Those would influence the secondary structure of the enzyme and change the spectra characteristics.
3.3.1. Circular Dichroism Spectra. The two negative peaks near 208 and $222 \mathrm{~nm}$ are the main characteristic peaks corresponding to the $\alpha$-helix structure of protein [17]. The $\mathrm{CD}$ spectra of lysozyme in the absence of luteolin or luteoloside (Figure 4) exhibited two negative peaks at 209 and $222 \mathrm{~nm}$, which was the characteristic peaks of pure lysozyme $\alpha$-helix. Although the two negative peaks were at 212 and $220 \mathrm{~nm}$ or 214 and $222 \mathrm{~nm}$ (Figure 4(B) and Figure $4(\mathrm{C})$ ), after adding luteolin or luteoloside, the CD spectra of lysozyme in the absence and presence of luteolin or luteoloside were similar in shape, suggesting the structure of lysozyme was also $\alpha$-helical predominantly. In terms of significant changes in intensity and position, we inferred the lysozyme $\alpha$-helix was influenced by the luteolin and luteoloside. Besides that, the two negative peaks intensities of lysozyme increased, indicating the increase of $\alpha$-helix content in lysozyme [30].

The obvious difference in the CD spectra, when luteolin or luteoloside was added into the lysozyme solution, reconfirmed the interaction between lysozyme and luteolin or luteoloside causing the changes in lysozyme $\alpha$-helix.

3.3.2. Raman Spectra. The three peaks [31] at 1653, 1296, and $1277 \mathrm{~cm}^{-1}$ in amide I band and amide III band are the main characteristic peaks of lysozyme $\alpha$-helix in Raman spectra. A, B, and C in Figure 5 exhibited the intensities and shifts of three characteristic peaks of lysozyme $\alpha$-helix changed after luteolin or luteoloside added, although the Raman spectra of lysozyme in the absence and presence of luteolin or luteoloside were similar in shape, from which we inferred the lysozyme $\alpha$-helix was influenced by luteolin or luteoloside.

3.3.3. ATR-FTIR Spectra. According to the above spectra, the lysozyme $\alpha$-helix structure might be influenced after adding luteolin and luteoloside. To further study the changes of lysozyme $\alpha$-helix relative content, the ATR-FTIR method was used to confirm the conjectures of CD and Raman spectra.

The absorption bands observed in the amide I region correspond to $\alpha$-helix $\left(1642-1660 \mathrm{~cm}^{-1}\right), \beta$-sheet $\left(1613-1637 \mathrm{~cm}^{-1}\right.$ and $\left.1682-1689 \mathrm{~cm}^{-1}\right), \beta$-turn $(1662-$ $\left.1700 \mathrm{~cm}^{-1}\right)$, and disordered $\left(1637-1645 \mathrm{~cm}^{-1}\right)$ structures [21].

The relative contents of lysozyme secondary structure in the absence and presence of luteolin and luteoloside are shown in Table 1. The correlative differential spectra and Gaussian fitting plots of lysozyme, (luteolin-LYSO)-luteolin, (luteoloside-LYSO)-luteoloside are shown in Figure 6, respectively.

The relative contents of lysozyme secondary structure were changed after adding luteolin and luteoloside. From Table 1, the average relative contents of lysozyme $\alpha$-helix in the presence of luteolin and luteoloside significantly increased by $43 \%$ and $30 \%$ with $\beta$-sheet decreasing by $19 \%$ and $14 \%$, respectively, indicating the interaction between lysozyme and luteolin or luteoloside induced conformational compactness of lysozyme 


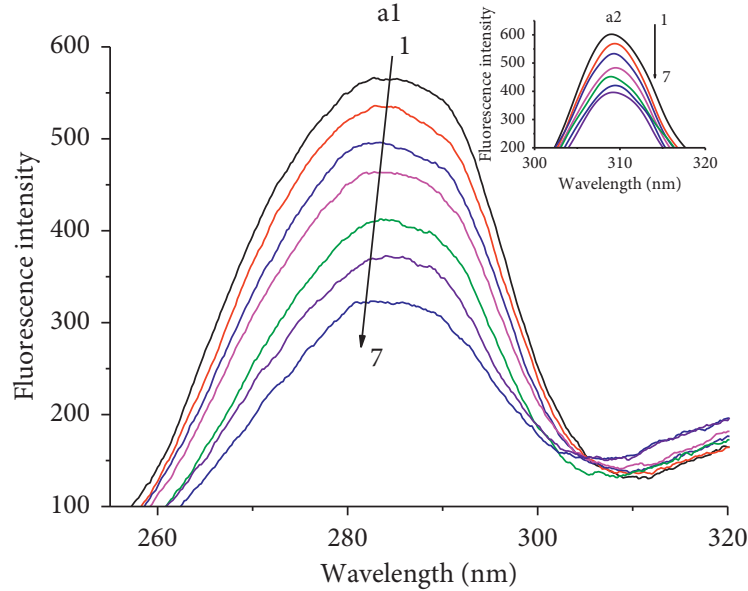

(a)

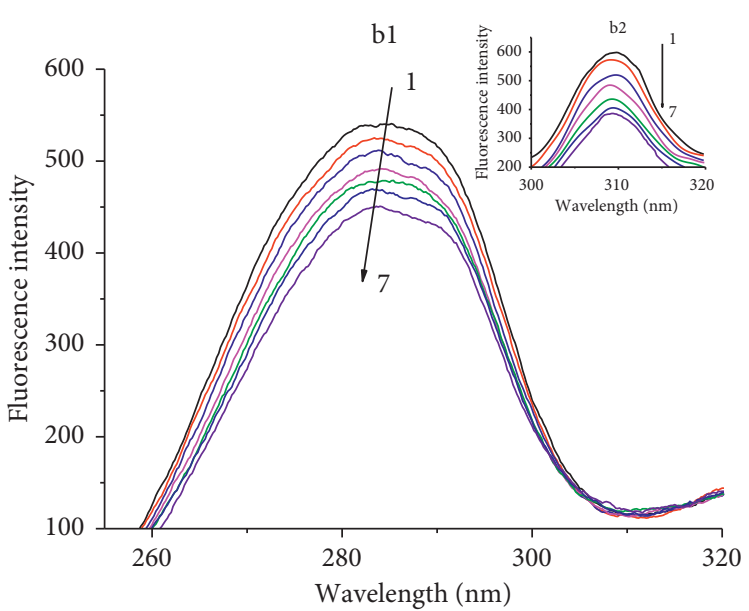

(b)

FIGURE 2: Synchronous fluorescence spectra of luteolin-LYSO (a1) and luteoloside-LYSO (b1) and fluorescence quenching spectra of luteolin-LYSO (a2) and luteoloside-LYSO(b2), when $\Delta \lambda=60 \mathrm{~nm}, T=298 \mathrm{~K}$. From curve $1 \longrightarrow 7, C_{(\mathrm{LYSO})}=1.0 \mu \mathrm{mol} / \mathrm{L}$, $C_{\text {(luteolin) }}=C_{\text {(luteoloside) }}=0,1.0,2.0,3.0,4.0,5.0$, and $6.0 \mu \mathrm{mol} / \mathrm{L}$, respectively.

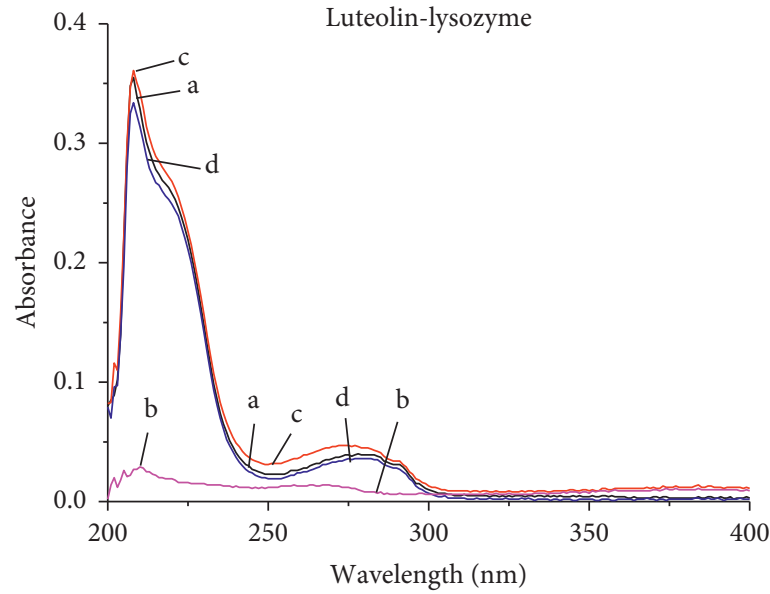

(a)

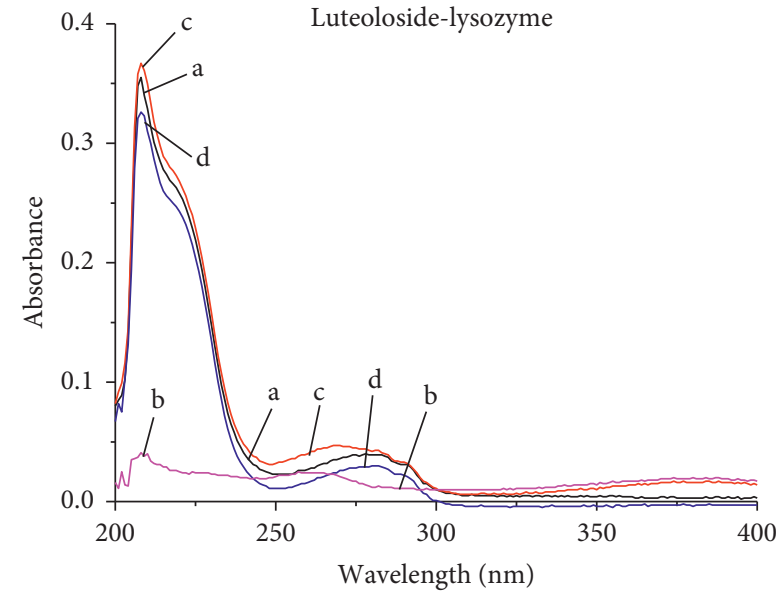

(b)

FIgURE 3: The absorption spectra of LYSO, luteolin (or luteoloside), and luteolin (or luteoloside)-LYSO system. (a) LYSO, (b) luteolin (or luteoloside), (c) luteolin (or luteoloside)-LYSO, and (d) luteolin (or luteoloside)-LYSO-luteolin (or luteoloside); $C_{(\text {LYSO })}=C_{(\text {luteolin })}=C_{(\text {luteoloside })}=1.0 \mu \mathrm{mol} / \mathrm{L}$.

peptides to increase hydrophobicity of microenvironment, which were coincident with the conclusions of synchronous fluorescence spectra.

Luteoloside had less effect on the lysozyme helices structure than that of luteolin did; maybe a molecule of glucose on the 7-hydroxyl group of luteoloside impeded the binding of luteoloside to lysozyme, suggesting the lysozyme secondary structure was influenced by the steric hindrance.

There was a significant difference in the lysozyme $\alpha$-helix relative contents after luteolin or luteoloside was added with $P$ values less than 0.05 , which indicated the active site of lysozyme and the biological function of lysozyme might be affected.
3.4. Lysozyme Activity. The change of lysozyme $\alpha$-helix relative content might cause the change of lysozyme active site, and assay kit was used to evaluate the activities of lysozyme in the absence and presence of luteolin and luteoloside. The lysozyme activity plots (Figure 7) were obtained. The lysozyme activity decreased obviously with the addition of luteolin and luteoloside. The change of lysozyme activity in the presence of luteolin was more obvious than that of luteoloside, suggesting the steric hindrance might also affect the lysozyme activity. The results indicated the changes of lysozyme helices structure were closely related to the changes of the activity. 


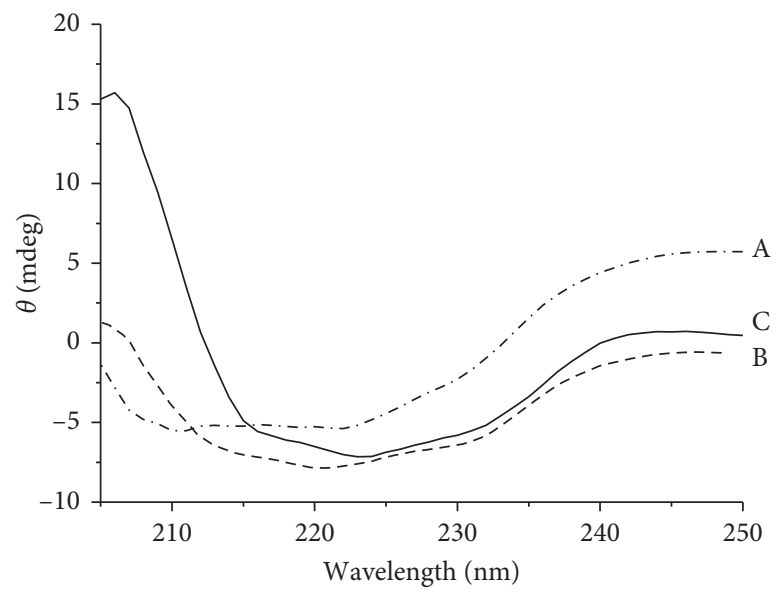

... A

-.- B

- C

FIgure 4: The CD spectra of (A) lysozyme, (B) lysozyme-luteolin, and (C) lysozyme-luteoloside. $C_{(\mathrm{LYSO})}=1 \times 10^{-2} \mathrm{mmol} / \mathrm{L}$, $C_{(\text {luteolin) }}=C_{\text {(luteoloside) }}=3 \times 10^{-1} \mathrm{mmol} / \mathrm{L}$.

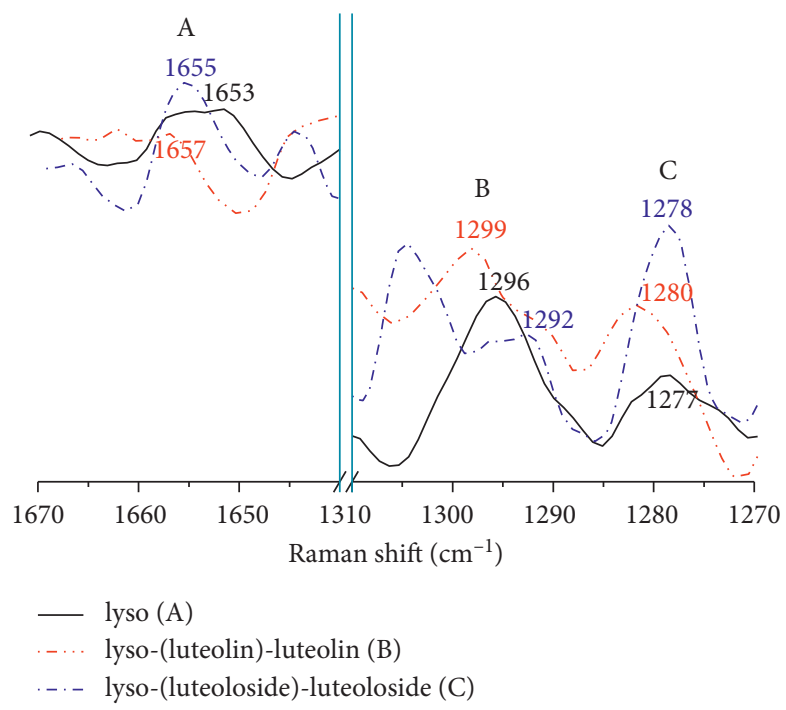

FIGURE 5: Raman spectra of LYSO (A), (luteolin-LYSO)-luteolin, (B) and (luteoloside-LYSO)-luteoloside $(\mathrm{C}) . C_{(\mathrm{LYSO})}=1 \times 10^{-2} \mathrm{mmol} / \mathrm{L}$, $C_{(\text {Luteolin })}=C_{(\text {Luteoloside })}=5 \times 10^{-2} \mathrm{mmol} / \mathrm{L}$.

TABLE 1: The relative contents of lysozyme secondary structure $(n=3)$.

\begin{tabular}{lcccc}
\hline & $\alpha$-Helix & $\beta$-Sheet & Disordered & $\beta$-Turn \\
\hline LYSO & $22.63 \pm 1.561$ & $32.54 \pm 1.056$ & $11.69 \pm 1.299$ & $33.13 \pm 0.933$ \\
(Luteolin-LYSO)-luteolin & $32.47 \pm 1.726^{*}$ & $26.33 \pm 2.718^{*}$ & $11.08 \pm 1.500$ & $30.12 \pm 1.218^{*}$ \\
(Luteoloside-LYSO)-luteoloside & $29.33 \pm 0.709^{*}$ & $27.80 \pm 3.300$ & $9.54 \pm 0.228$ & $33.32 \pm 3.993$ \\
\hline
\end{tabular}

${ }^{*} P<0.05$ compared with lysozyme system. 


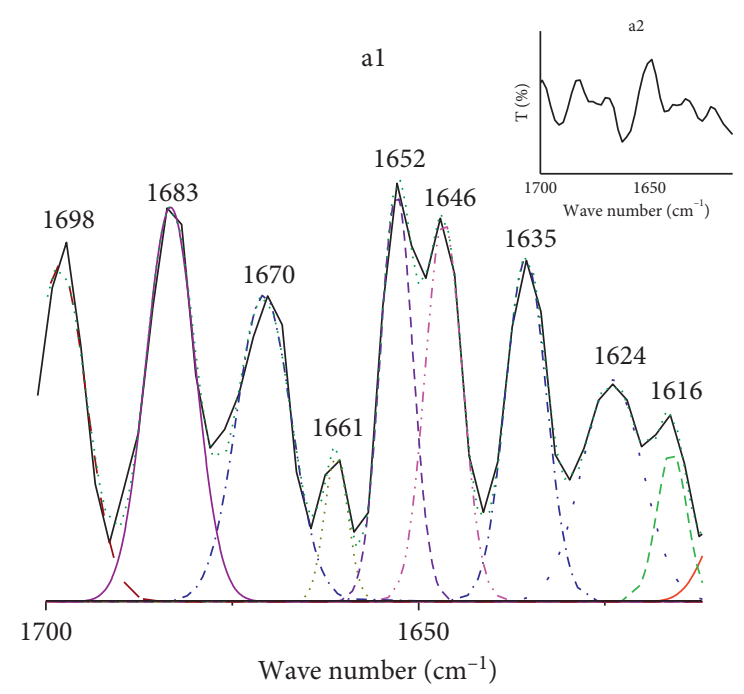

(a)

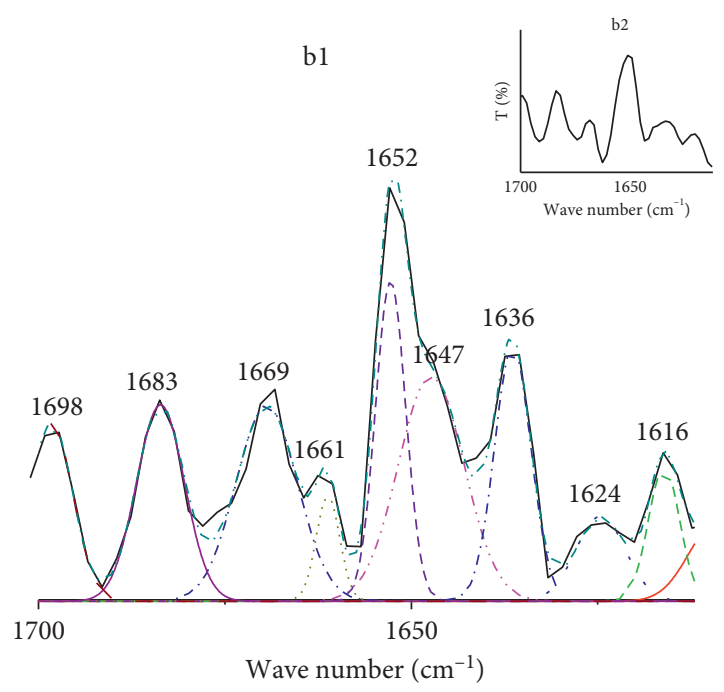

(b)

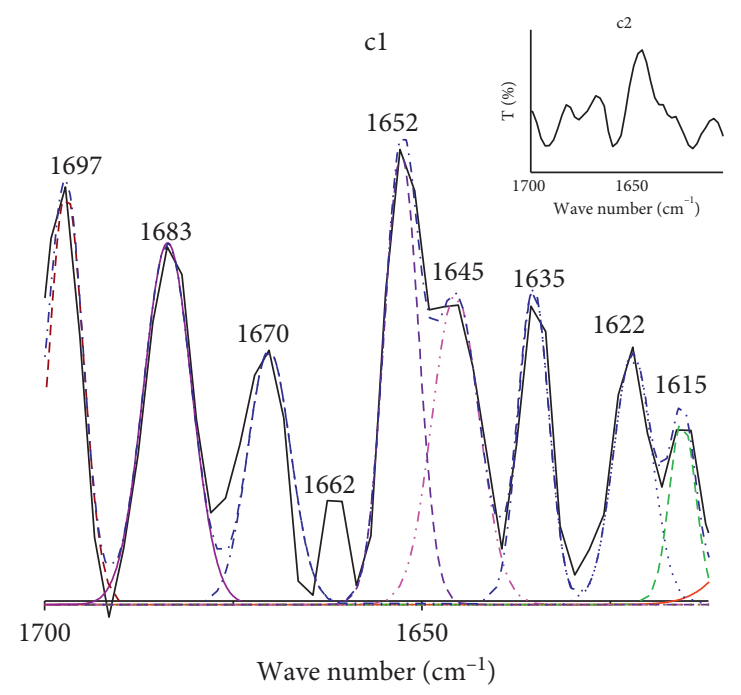

(c)

FIGURE 6: ATR-FTIR curve-fitting spectra of LYSO (a1), (luteolin-LYSO)-luteolin (b1) and (luteoloside-LYSO)-luteoloside (c1), and differential spectra of LYSO (a2), (luteolin-LYSO)-luteolin (b2), and (luteoloside-LYSO)-luteoloside (c2), when T=298K. $C_{(\mathrm{LYSO})}=1 \times 10^{-2} \mathrm{mmol} / \mathrm{L}, C_{(\text {luteolin })}=C_{(\text {luteoloside })}=5 \times 10^{-2} \mathrm{mmol} / \mathrm{L}$, respectively.

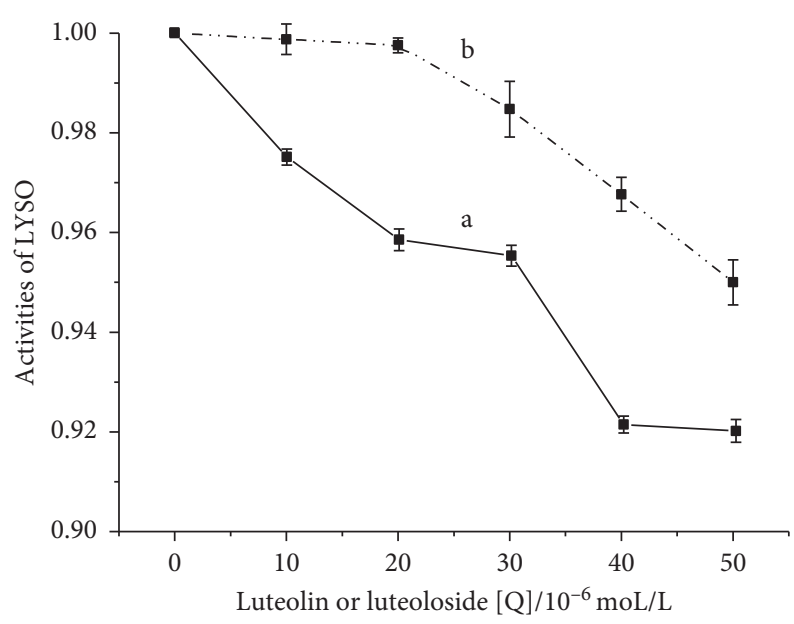

FIgURE 7: The activities of LYSO at different concentrations of luteolin or luteoloside. (a) luteolin-LYSO and (b) luteoloside-LYSO. 


\section{Conclusions}

Fluorescence and UV spectra analyses proved that luteolin and luteoloside could combine with lysozyme. According to the ATR-FTIR method, the lysozyme $\alpha$-helix relative content had a significant difference in the absence and presence of luteolin and luteoloside, which confirmed the conjectures of fluorescence spectra, CD spectra, and Raman spectra. Lysozyme activity experiment indicated the activities of lysozyme relating to $\alpha$-helix structure and the more the $\alpha$-helix changed, the more the biological activity was affected. The results might provide important theoretical research for the attempt of combined utilization between lysozyme and luteolin or luteoloside in the field of food and health.

\section{Data Availability}

The data used to support the findings of this study are included within the article.

\section{Disclosure}

Lijian Cui is the co-first author.

\section{Conflicts of Interest}

The authors declare that they have no conflicts of interest.

\section{Authors' Contributions}

$\mathrm{Yu} \mathrm{Wu}$ and Lijian Cui contributed equally to this work.

\section{Acknowledgments}

This work was supported by the National Key Research Project of China (2019YFC1606400), National Key Research Project of Hebei Province (20375502D), Natural Science Foundation of Hebei Province (H2017206281, H2019206535, H2020206003), High-Level Talent Funding Project of Hebei Province (A201905006), Science and Technology Research Project of Hebei Province (QN2018058), and Fund of National R\&D Center for Edible Fungus Processing Technology, Henan University (20200109).

\section{References}

[1] T. Wu, Q. Jiang, D. Wu et al., "What is new in lysozyme research and its application in food industry? a review," Food Chemistry, vol. 274, pp. 698-709, 2019.

[2] S. A. Hu and A. K. Criss, "From bacterial killing to immune modulation: recent insights into the functions of lysozyme," PLoS Pathogens, vol. 13, no. 9, Article ID e1006512, 2017.

[3] Y. Kawai, K. Mickiewicz, and J. Errington, "Lysozyme counteracts $\beta$-lactam antibiotics by promoting the emergence of L-form bacteria," Cell, vol. 172, no. 5, pp. 1038-1049, 2018.

[4] Y. Yang, Y. Sun, N. Zhang et al., "The up-regulation of two identified wound healing specific proteins-HSP70 and lysozyme in regenerated Eisenia fetida through transcriptome analysis," Journal of Ethnopharmacology, vol. 237, pp. 64-73, 2019.
[5] R. M. Li, D. T. Ali, R. G. Mohammad, and B. M. Ahmad, "Structural-functional integrity of lysozyme in imidazolium based surface active ionic liquids," International Journal of Biological Macromolecules, vol. 156, pp. 271-279, 2020.

[6] S. Das, Z. Hazarika, S. Sarmah et al., "Exploring the interaction of bioactive kaempferol with serum albumin, lysozyme and hemoglobin: a biophysical investigation using multispectroscopic, docking and molecular dynamics simulation studies," Journal of Photochemistry and Photobiology B: Biology, vol. 205, Article ID 111825, 2020.

[7] S. Baruah, L. Perez De Souza, M. Benina, and A. R. Fernie, "The style and substance of plant flavonoid decoration; towards defining both structure and function," Phytochemistry, vol. 174, Article ID 112347, 2020.

[8] S. Wang, L. Fu, Y. Wu, H. Xiao, J. Wang, and G. Sun, "Influence of luteolin on the apoptosis of esophageal cancer Eca109 cells and its mechanism of action," Food Science and Human Wellness, vol. 8, no. 2, pp. 189-194, 2019.

[9] Y. Huang, L.-J. Cui, J.-M. Wang et al., "Comparative studies on interactions of baicalein, baicalin and scutellarin with lysozyme," European Journal of Medicinal Chemistry, vol. 46, no. 12 , pp. 6039-6045, 2011.

[10] A. Huo, "Analysis of various solid samples by synchronous fluorescence spectroscopy and related methods: a review," Talanta, vol. 216, Article ID 120944, 2020.

[11] K. Wang, D.-W. Sun, H. Pu, and Q. Wei, "Principles and applications of spectroscopic techniques for evaluating food protein conformational changes: a review," Trends in Food Science \& Technology, vol. 67, pp. 0924-2244, 2017.

[12] L. Dimitrova-Paternoga, P. K. A. Jagtap, P.-C. Chen, and J. Hennig, "Integrative structural biology of protein-RNA complexes," Structure, vol. 28, no. 1, pp. 6-28, 2020.

[13] S. M. Kelly, T. J. Jess, and N. C. Price, "How to study proteins by circular dichroism," Biochimica et Biophysica Acta (BBA)Proteins and Proteomics, vol. 1751, no. 2, pp. 119-139, 2005.

[14] C. M. Laurent, J. M. Dyke, R. B. Cook, G. Dyke, and R. de Kat, "Spectroscopy on the wing: investigating possible differences in protein secondary structures in feather shafts of birds using Raman spectroscopy," Journal of Structural Biology, vol. 211, no. 1, Article ID 107529, 2020.

[15] G. Bellocco, "FTIR, ESI-MS, VT-NMR and SANS study of trehalose thermal stabilization of lysozyme," International Journal of Biological Macromolecules: Structure, Function and Interactions, vol. 63, pp. 225-232, 2014.

[16] H. Tiernan, B. Byrne, and S. G. Kazarian, "ATR-FTIR spectroscopy and spectroscopic imaging for the analysis of biopharmaceuticals," Spectrochimica Acta Part A: Molecular and Biomolecular Spectroscopy, vol. 241, Article ID 118636, 2020.

[17] D. M. Rogers, S. B. Jasim, N. T. Dyer, F. Auvray, and M. Réfrégiers, "Electronic circular dichroism spectroscopy of proteins," Chem, vol. 5, no. 11, pp. 2751-2774, 2019.

[18] M. Hirst, C. Hao, Z. Qing et al., "Protective characterization of low dose sodium nitrite on yak meat myoglobin in a hydroxy radical oxidation environment: Fourier transform Infrared spectroscopy and laser Micro-Raman spectroscopy," Academic Press, vol. 116, Article ID 108556, 2019.

[19] Z. Q. Wen, "Raman spectroscopy of protein pharmaceuticals," Journal of Pharmaceutical Sciences, vol. 96, no. 11, pp. 2861-2878, 2007.

[20] Z. Ming, N. Yingqun, A. Paul, and D. Gerard, "Application of Raman spectroscopy and chemometric techniques to assess sensory characteristics of young dairy bull beef," Food Research International, vol. 107, pp. 27-40, 2018. 
[21] L. Sheng, J. Wang, M. Huang, Q. Xu, and M. Ma, "The changes of secondary structures and properties of lysozyme along with the egg storage," International Journal of Biological Macromolecules, vol. 92, pp. 600-606, 2016.

[22] B. Mandal, S. Mondal, A. Pan, S. P. Moulik, and S. Ghosh, "Physicochemical study of the interaction of lysozyme with surface active ionic liquid 1-butyl-3-methylimidazolium octylsulfate [BMIM] [OS] in aqueous and buffer media," Colloids and Surfaces A: Physicochemical and Engineering Aspects, vol. 484, pp. 0927-7757, 2015.

[23] R. K. Mishra, A. Hayat, G. K. Mishra, G. Catanante, V. Sharma, and J. L. Marty, "A novel colorimetric competitive aptamer assay for lysozyme detection based on superparamagnetic nanobeads," Talanta, vol. 165, pp. 436-441, 2016.

[24] S. Yvon, L. Schwebel, L. Belahcen et al., "of thermized donkey milk with lysozyme activity on altered gut barrier in mice exposed to water-avoidance stress," Journal of Dairy Science, vol. 102, no. 9, pp. 7697-7706, 2019.

[25] T. G. Haimoud-Lekhal and J. Popp, "Electric field standing wave effects in internal reflection and ATR spectroscopy," Spectrochimica Acta Part A: Molecular and Biomolecular Spectroscopy, vol. 191, pp. 165-171, 2018.

[26] G. Güler, M. M. Vorob'ev, V. Vogel, and W. Mäntele, "Proteolytically-induced changes of secondary structural protein conformation of bovine serum albumin monitored by Fourier transform infrared (FT-IR) and UV-circular dichroism spectroscopy," Spectrochimica Acta Part A: Molecular and Biomolecular Spectroscopy, vol. 161, pp. 8-18, 2016.

[27] A. Fl, B. Mm, A. Jr et al., "Photo-oxidation of lysozyme triggered by riboflavin is $\mathrm{O}_{2}$-dependent, occurs via mixed type 1 and type 2 pathways, and results in inactivation, site-specific damage and intra- and inter-molecular crosslinks," Free Radical Biology and Medicine, vol. 152, pp. 61-73, 2020.

[28] D. Juretić, Y. Sonavane, N. Ilić et al., "Designed peptide with a flexible central motif from ranatuerins adapts its conformation to bacterial membranes," Biochimica et Biophysica Acta-Biomembranes, vol. 1860, no. 12, pp. 2655-2668, 2018.

[29] N. Zoranić, D. D. Mulmi, and M. L. Nakarmi, "Optical transitions in lysozyme mediated zinc oxide nanoparticles probed by deep UV photoluminescence," Optik, vol. 202, Article ID 163622, 2020.

[30] J. M. Khan, A. Ahmed, S. Farah et al., "Millimolar concentration of sodium dodecyl sulfate inhibit thermal aggregation in hen egg white lysozyme via increased $\alpha$-helicity," Colloids and Surfaces A: Physicochemical and Engineering Aspects, vol. 572, pp. 167-173, 2019.

[31] X. Hussain, L. Yang, Y. Li, E. C. Cheshari, and X. Li, "The integration of molecular imprinting and surface-enhanced Raman scattering for highly sensitive detection of lysozyme biomarker aided by density functional theory," Spectrochimica Acta Part A: Molecular and Biomolecular Spectroscopy, vol. 228, Article ID 117764, 2020. 